\title{
CONTROL OF OSCILLATIONS AND STATE ESTIMATION OF KAPITZA PENDULUM ${ }^{1}$
}

\author{
Iliya V. Miroshnik and Nikolay M. Odinets
}

\author{
Lab. "Control of Complex Systems", Institute for Problems \\ of Mech. Eng. of Russian Academy of Sciences \\ Lab. of Cybernetics and Control Systems, State University \\ of Information Technologies, Mechanics and Optics \\ Sablinskaya 14, Saint-Petersburg, 197101 Russia \\ miroshnik@yandex.ru; nmodinets@yandex.ru
}

\begin{abstract}
In this paper we introduce the concept of virtual energy of Kapitza pendulum and propose an energy-based approach to problem of stabilization of the given periodic motions around the upright position. The problem is reduced to control of the virtual energy and implies estimating the pendulum's slow motions provided by a nonlinear observer of the slow oscillations and virtual energy. New simulation results are represented. Copyright (C)IFAC 2005.
\end{abstract}

Keywords: robotics, nonlinear control, pendular systems, virtual energy, oscillation stabilization.

\section{INTRODUCTION}

Problems of stabilization of oscillating motions in pendular systems are solved by using horizontal movement of the pivot (Chung and Hauser, 1995; Andrievsky et al., 1996; Fradkov, 1996; Fradkov and Pogromsky, 1998; Shiriaev et al., 1998; Miroshnik and Bobtzov, 2000; Aracil et al., 2002) or its vertical displacement (Fradkov et al., 1999; Miroshnik and Olkhovskaya, 2003). Known control laws provide periodic motions of the pendulums in the neighborhood of the lower position. Nevertheless, under a high-frequency vertical excitation of the pendulum support, one can observe a complex periodic motion of the pendulum in the vicinity of the upright position referred to as induced or vibrational stability. The inverted pendulum on vibrating support known as pendulum of Kapitza was studied in a great number of scien-

\footnotetext{
1 This work is supported by Russian Foundation for Basic Research and the Presidium of RAS (program N 19 "Control of mechanical systems", project 1.4).
}

tific publications (see (Stephenson, 1908; Kapitza, 1951; Bogolyubov and Mitropolsky, 1962; Belman et al., 1986; Blekhman, 1988; Yabuno et al., 2004)) and represents a considerable interest of the researchers as a good benchmarks for many natural, technological and physical phenomena from biological processes and vibrational technologies to problems of anti-gravity.

Stability of the upper equilibrium point of free (uncontrollable and conservative) model of Kapitza pendulum leads to undamped low-frequency oscillations of the pendulum around upright position. However these oscillations are unstable and their amplitudes depend on the pendulum initial state. Stability of a given periodic motion of the pendulum can be archived by using an auxiliary vertical motion of the support and the energybased control developed for "normal" pendulum with vertically moved support (Fradkov et al., 1999; Miroshnik and Olkhovskaya, 2003; Miroshnik and Odinets, 2004). The main difficulties of this solution are connected with separating in- 
formation about slow motion of the pendulum (Odinets and Levidova, 2004).

In this paper we introduce the concept of virtual energy of Kapitza pendulum associated with energy of slow motion of an inverted pendulum with opposite gravity direction and propose an energybased approach to the problem of stabilization of the given periodic motions. The problem is reduced to control of the virtual energy and implies estimating the pendulum's slow motions provided by a nonlinear observer of the slow oscillations and virtual energy. New simulation results are represented to confirm the main theoretical aspects of the solution.

The paper is organized as follows. Models of pendulum with vertical vibration of the pivot and their properties are represented in Section 2. In Section 3, we introduce the virtual energy of Kapitza pendulum, derive a model of the slow motion and, on this basis, design a nonlinear observer of the energy. In Section 4 the problem of control of Kapitza pendulum oscillations is solved as that of stabilization of the virtual energy by using an appropriate nonlinear observer.

\section{MODELS OF PENDULA AND PIVOT EXCITATION}

(a)
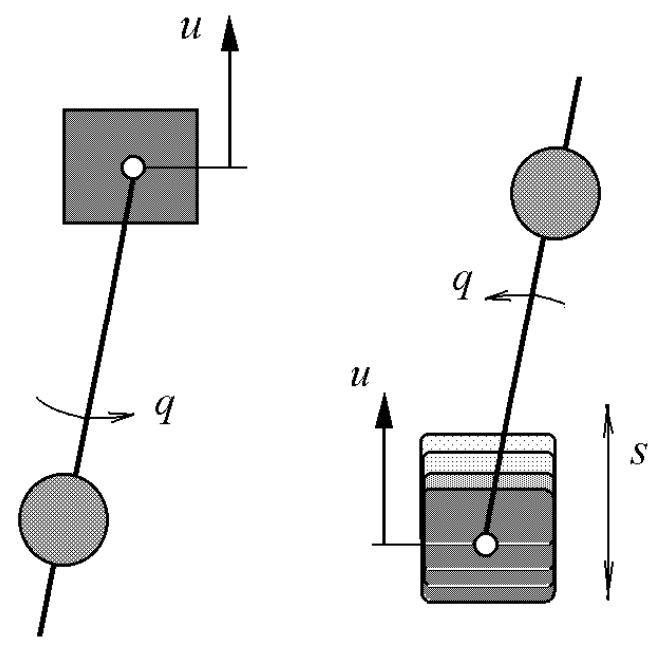

Fig. 1. Pendula with mobile supports

We consider a model of a pendulum on the mobile support written in the Hamiltonian form

$$
J \dot{q}=p, \quad \dot{p}=-\frac{\partial \Pi}{\partial q}+G(q) u,
$$

where $q$ is the generalized coordinate (angle), $p=J \dot{q}$ is the momentum, $u$ is the control action,
$J=m l^{2}, \Pi(q)$ is the potential energy, $G(q)$ is a function. The total energy (Hamiltonian function) of the unforced pendulum is computed as

$$
E(q, p)=\Pi(q)+T(p)=\Pi(q)+\frac{1}{2 J} p^{2},
$$

where $T(p)$ is the kinetic energy.

When the pivot accomplishes vertical motion (see Fig.1,a),

$$
\begin{gathered}
\Pi(q)=m g l(1-\cos q), \\
G(q)=-m l \sin q,
\end{gathered}
$$

and equation (1) takes the form

$$
J \dot{q}=p, \quad \dot{p}=-m g l \sin q-m l \sin q u .
$$

Problems of control of oscillations are usually associated with keeping up a required mode of the undamped periodic pendulum motion. Taking into account that the mode of oscillations is connected with a certain level of pendulum internal energy (Andrievsky et al., 1996; Fradkov and Pogromsky, 1998; Fradkov et al., 1999), the problems are reduced to those of energy stabilization. The latter is a standard nonlinear problem of partial stabilization of a dynamical system, or stabilization with respect to the function $E=E(q, p)$ (Fradkov et al., 1999).

It is worth to note that the unforced pendulum (3) has two equilibrium points. The first one $(q, p)=(0,0)$ corresponds to the lower position of the pendulum and is asymptotically stable. The other points $(q, p)=( \pm \pi, 0)$ associated with the upright position, are unstable and, in the case considered, cannot be stabilized by using standard control techniques. The same situation is observed in pendulum oscillations. Energy-based control provides stable oscillation around the lower point, corresponding to a given energy level

$$
E^{*}<E_{m}=2 m g l .
$$

If $E^{*}>E_{m}$, the pendulum demonstrates proportional rotation around the pivot. Oscillations of the pendulum around the upper position are impossible without special pivot excitation.

High-frequency vertical vibration of the support (see Fig. 1b) essentially changes properties of the pendulum (Stephenson, 1908; Kapitza, 1951; Bogolyubov and Mitropolsky, 1962; Belman et al., 1986; Blekhman, 1988; Yabuno et al., 2004). Under the relevant conditions, the open loop pendular system known as Kapitza pendulum becomes stable (or asymptotically stable) with respect to the upper equilibriums $(q, p)=( \pm \pi, 0)$. This property is a crucial point to the problem of the control of pendulum oscillations around the upright position. 
Consider a pendulum under a high-frequency vertical excitation of the pivot, when the coordinate of the support $s$ is described by the equation

$$
\ddot{s}+\omega^{2} s=0
$$

where $s(0)=s_{0}, \dot{s}(0)=\dot{s}_{0}, \omega$ is a frequency of vibration, or

$$
s(t)=A \sin (\omega t+\varphi),
$$

where $A=A\left(s_{0}, \dot{s}_{0}\right), \varphi=\varphi\left(s_{0}, \dot{s}_{0}\right)$. This mode is provided by the control

$$
u=\ddot{s}=-\omega^{2} s .
$$

Substituting (5) into the model (3), one obtains

$$
J \dot{q}=p, \quad \dot{p}=-m l\left(g-\omega^{2} s\right) \sin q .
$$

If the frequency $\omega$ is large enough and the amplitude is relatively small, the solution of system (6) is approximately represented by a two-frequency signal of the form (Bogolyubov and Mitropolsky, 1962; Blekhman, 1988)

$$
q \cong \bar{q}-\widetilde{s} \sin \bar{q}
$$

Here

$$
\widetilde{s}(t)=\frac{m l}{J}\left(s-s_{0}\right)
$$

is a fast component of the oscillations, and $\bar{q}(t)$ is a slow component, being, in turn, a solution of the equations

$$
J \dot{\bar{q}}=\bar{p}, \quad \dot{\bar{p}}=-m\left(g l+\frac{\theta \omega^{2} A^{2}}{2}\right) \sin \bar{q},
$$

where

$$
\theta=1 \text { if }|\bar{q}|<\frac{\pi}{2}, \quad \theta=-1 \text { if } \frac{\pi}{2}<|\bar{q}|<\frac{3 \pi}{2} .
$$

When

$$
\omega^{2} A^{2}>2 g l
$$

equation of slow motion (9) can be rewritten as

$$
J \dot{\bar{q}}=\bar{p}, \quad \dot{\bar{p}}=-\theta m l^{2} \bar{\omega}^{2} \sin \bar{q},
$$

where

$$
\bar{\omega}=\sqrt{\frac{2 g \theta l+\omega^{2} A^{2}}{2 l^{2}}},
$$

or in the form

$$
\ddot{\bar{q}}+\theta \bar{\omega}^{2} \sin \bar{q}=0 .
$$

The latter shows that the system acquires two stable equilibriums $(q, p)=(0,0)$ and $(q, p)=$ $( \pm \pi, 0)$, and the pendulum can accomplish "slow"
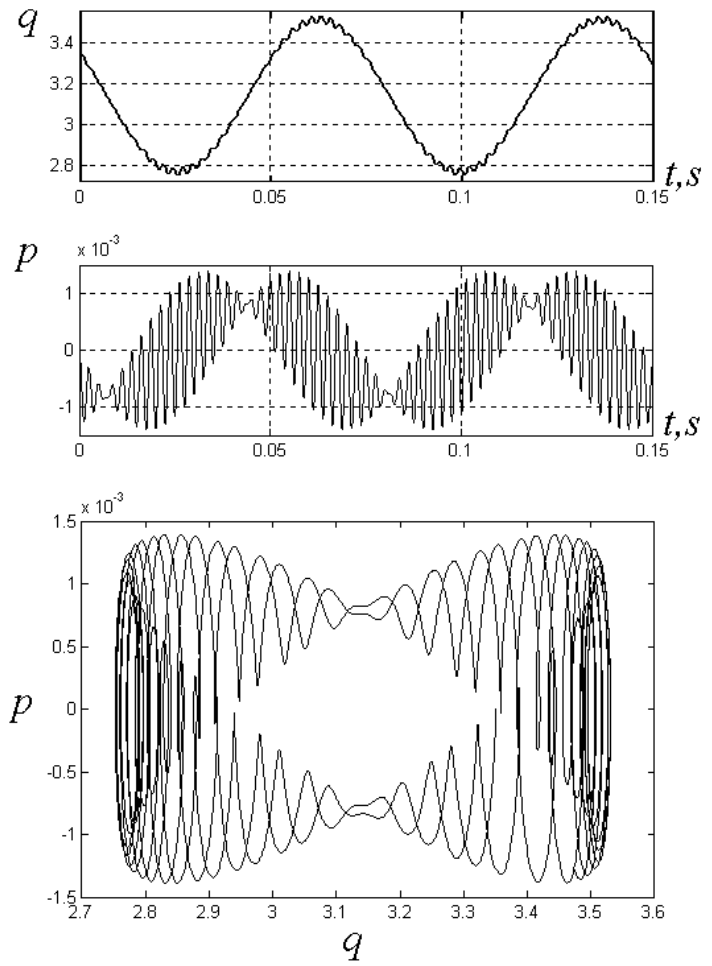

Fig. 2. Free oscillations around upper position oscillations around the upper (Fig. 2) or lower positions.

Thus, under the high-frequency excitation of the pivot, the equilibrium points $(q, p)=( \pm \pi, 0)$ become stable that enable one, by using appropriate control actions, to provide the required stable slow oscillations of the pendulum around the upright position.

\section{VIRTUAL ENERGY AND NONLINEAR OBSERVER}

The problem of stabilization of Kapitza pendulum oscillation around upper position can be reduced to that of energy stabilization. This implies the use of a concept of virtual energy (Miroshnik and Odinets, 2004) and estimation of the slow motion variables $\bar{q}$ and $\bar{p}$.

Consider the Kapitza pendulum motion in a neighborhood of the upright position, where $\pi / 2<|\bar{q}|<3 \pi / 2$ and $\theta=-1$. Introduce virtual gravity acceleration

$$
\bar{g}=\frac{\omega^{2} A^{2}}{2 l}-g>0
$$

turned to the opposite direction with respect to the gravity, and virtual energy of slow motion as

$$
\bar{E}(\bar{q}, \bar{p})=\bar{\Pi}(\bar{q})+\frac{1}{2 J} \bar{p}^{2},
$$

where

$$
\bar{\Pi}(\bar{q})=m \bar{g} l(1+\cos \bar{q}) .
$$


is the virtual potential energy. Note that $\bar{\Pi}(\bar{q}) \geq 0$ in the neighborhood of the upright position and $\bar{\Pi}( \pm \pi)=0$. Then the model of the pendulum system (11) can be rewritten as

$$
J \dot{\bar{q}}=\bar{p}, \quad \dot{\bar{p}}=-\frac{\partial \bar{\Pi}}{\partial \bar{q}} .
$$

The latter is equivalent to a description of the free motion of the ordinary pendulum (see (1) for $u=0)$.

In order to estimate the current value of the virtual energy (14), it is necessary to separate the signals $\bar{q}$ and $\bar{p}$ from the measurable two-frequency signal $q$. This is realized by using the nonlinear observer, the structure of which is similar to the pendulum model (16),(7) (see also (Odinets and Levidova, 2004)):

$$
\begin{aligned}
J \dot{\bar{q}}_{e} & =\bar{p}_{e}+u_{e 1}, \quad \dot{\bar{p}}_{e}=-\frac{\partial \bar{\Pi}}{\partial \bar{q}_{e}}+u_{e 2}, \\
q_{e} & =\bar{q}_{e}-\widetilde{s} \sin \bar{q}_{e}
\end{aligned}
$$

where $\bar{q}_{e}, \bar{p}_{e}, q_{e}$ are the estimates of the relevant variables, $u_{e 1}, u_{e 2}$ are the observer feedback signals to be found.

Introduce the errors of the observer (residuals)

$$
\begin{aligned}
\widetilde{q}_{e} & =\bar{q}-\bar{q}_{e}, \quad \widetilde{p}_{e}=\bar{p}-\bar{p}_{e}, \\
\widetilde{q} & =q-q_{e}
\end{aligned}
$$

and note that the error $\widetilde{q}$ is measurable and can be used for feedback design. For small enough errors, one can obtain the linearized error model

$$
\begin{aligned}
J \dot{\tilde{q}}_{e} & =\widetilde{p}_{e}-u_{e 1}, \quad \dot{\tilde{p}}_{e}=-\alpha\left(\bar{q}_{e}\right) \widetilde{q}_{e}-u_{e 2} \\
\widetilde{q} & =(1-\widetilde{s}) \widetilde{q}_{e}
\end{aligned}
$$

where

$$
\alpha\left(\bar{q}_{e}\right)=\frac{\partial^{2} \bar{\Pi}\left(\bar{q}_{e}\right)}{\partial \bar{q}_{e}^{2}} .
$$

Then, taking into the account that, for small $A$, $|s|<1$, we choose the observer feedbacks as

$$
\begin{aligned}
& u_{e 1}=J \frac{k_{1}}{1-\widetilde{s}} \widetilde{q}, \\
& u_{e 2}=-\alpha\left(\bar{q}_{e}\right) \widetilde{q}_{e}+J \frac{k_{2}}{1-\widetilde{s}} \widetilde{q},
\end{aligned}
$$

where $k_{1}>0, k_{2}>0$ are the feedback gains. It is easy to check that the choice of the feedbacks leads to the linear error model

$$
\ddot{\bar{q}}_{e}+k_{1} \dot{\bar{q}}_{e}+k_{2} \bar{q}_{e}=0 .
$$

The latter shows that $\bar{q}_{e} \rightarrow 0$ as $t \rightarrow \infty$, and, therefore, proves the asymptotic convergence of the estimates $\bar{q}_{e}, \bar{p}_{e}$ to the real values $\bar{q}, \bar{p}$.
Now, the require estimate of the virtual energy is found as

$$
\bar{E}_{e}=\bar{E}\left(\bar{q}_{e}, \bar{p}_{e}\right)=\bar{\Pi}\left(\bar{q}_{e}\right)+\frac{1}{2 J} \bar{p}_{e}^{2},
$$

where

$$
\bar{\Pi}\left(\bar{q}_{e}\right)=m \bar{g} l\left(1+\cos \bar{q}_{e}\right),
$$

and $\bar{E}_{e} \rightarrow \bar{E}$ as $t \rightarrow \infty$.

The validity of the result is confirmed by simulation. The pendulum with parameters $m=$ $0.005 \mathrm{~kg}, l=5 \mathrm{~cm}$ and pivot vibration $s=$ $0.25 \sin 400 t(\mathrm{~cm})$ is considered. Fig. 3 illustrates the perfect convergence of the estimates of slow processes of pendular system $\bar{q}_{e}, \bar{p}_{e}$ and $\bar{E}_{e}$ to the real values $\bar{q}(t), \bar{p}(t)$ and $\bar{E}$.
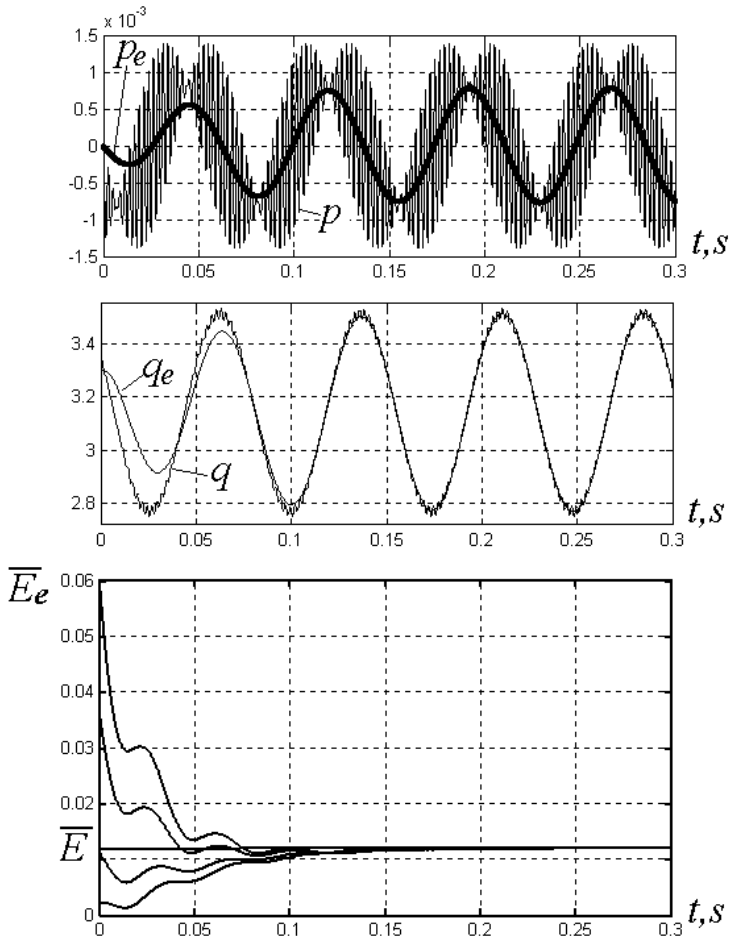

Fig. 3. Estimation of slow motions

\section{STABILIZATION OF OSCILLATIONS OF INVERTED PENDULUM}

Now, consider the controlled motion of the vibrating pendulum with respect to the upright position, supposing that condition (10) is satisfied. Such a motion is provided by the control

$$
u=\ddot{s}+\bar{u},
$$

where $\bar{u}$ is the stabilizing signal. The model of slow motion of the pendulum in the neighborhood of the upright position $\pi / 2<|q|<3 \pi / 2$ takes the form

$$
J \dot{\bar{q}}=\bar{p}, \quad \dot{\bar{p}}=m l \bar{g} \sin \bar{q}-m l \sin \overline{q u} .
$$


We introduce the virtual energy of the pendulum in the form (14) and rewrite the model (28) as

$$
J \dot{\bar{q}}=\bar{p}, \quad \dot{\bar{p}}=-\frac{\partial \bar{\Pi}}{\partial \bar{q}}+G(\bar{q}) \bar{u} .
$$

where

$$
G(\bar{q})=-m l \sin \bar{q} .
$$

Such as a desired mode of pendulum oscillations is associated with a certain level of its virtual energy, the control problem is reduced to that of energy stabilization mentioned in Section 2. The latter is a standard nonlinear problem of partial stabilization of a dynamical system, or stabilization with respect to the function $\bar{E}=$ $\bar{E}(\bar{q}, \bar{p})$ (Fradkov et al., 1999).

Let us set a desired energy level $\bar{E}^{*}$ and introduce the energy error (deviation)

$$
\xi=\bar{E}(\bar{q}, \bar{p})-\bar{E}^{*} .
$$

After simple manipulations, we obtain the error model

$$
\dot{\xi}=\frac{1}{J} \bar{p} G(\bar{q}) \bar{u}
$$

A stable solution of the problem is given by different control laws of the form

$$
\bar{u}=-J U(\bar{p}, G(\bar{q})) k \xi
$$

where $k>0$ is a feedback gain. Substituting (32) into (31), one obtains

$$
\dot{\xi}=-\bar{p} G U(\bar{p}, G(\bar{q})) k \xi .
$$

We can conclude that the system is asymptotically stable with respect to the given partial equilibrium point $\bar{E}=\bar{E}^{*}$ when the function $U(\bar{p}, G)$, for all $t>0$, satisfies the inequality

$$
\int_{0}^{t} \bar{p} G U(\bar{p}, G(\bar{q})) d \tau \geq \lambda t,
$$

where $\lambda>0$. It is easily seen, for instance, that the problem is solved by the stabilizing control laws

$$
\begin{aligned}
& \bar{u}=-J \operatorname{sign}(\bar{p} G) k \xi, \\
& \bar{u}=-J \bar{p} G k \xi .
\end{aligned}
$$

In order to estimate the current value of the virtual energy, a nonlinear observer of slow motion is designed by analogy with that of represented in Section 3. Taking into the account the structure of the system (29), we design the observer in the form

$$
\begin{aligned}
J \dot{\bar{q}}_{e} & =\bar{p}_{e}+u_{e 1} \\
\dot{\bar{p}}_{e} & =-\frac{\partial \bar{\Pi}}{\partial \bar{q}_{e}}+G\left(\bar{q}_{e}\right) \bar{u}+u_{e 2} \\
q_{e} & =\bar{q}_{e}-\widetilde{s} \sin \bar{q}_{e} .
\end{aligned}
$$

The observer feedbacks are chosen as

$$
\begin{aligned}
& u_{e 1}=J \frac{k_{1}}{1-\widetilde{s}} \widetilde{q} \\
& u_{e 2}=-\alpha\left(\bar{q}_{e}\right) \widetilde{q}_{e}+\beta\left(\bar{q}_{e}\right) \widetilde{q}_{e} \bar{u}+J \frac{k_{2}}{1-\widetilde{s}} \widetilde{q},
\end{aligned}
$$

where

$$
\beta\left(\bar{q}_{e}\right)=\frac{\partial G\left(\bar{q}_{e}\right)}{\partial \bar{q}_{e}},
$$

$k_{1}>0, k_{2}>0$. It is easy to check that the choice of the feedback leads to the error model (24) that proves the asymptotic convergence of the estimates $\bar{q}_{e}, \bar{p}_{e}$ to the real values $\bar{q}, \bar{p}$.

Then the require estimate of the virtual energy is found in the form (25)-(26) and the control is formed as

$$
\bar{u}=-J U\left(\bar{p}_{e}, G\left(\bar{q}_{e}\right) k \xi_{e},\right.
$$

where

$$
\xi_{e}=\bar{E}\left(\bar{q}_{e}, \bar{p}_{e}\right)-\bar{E}^{*}
$$

The validity of the result is confirmed by simulation. The pendulum with parameters $m=$ $0.005 \mathrm{~kg}, l=5 \mathrm{~cm}$ and pivot vibration $s=$ $0.25 \sin 400 t(\mathrm{~cm})$ is considered. The observerbased control law

$$
\bar{u}=J \operatorname{sign}\left(\bar{p}_{e} \sin \bar{q}_{e}\right) k \xi_{e}
$$

provides stabilization of the virtual energy $\bar{E}$ at the levels $\bar{E}^{*}$ from 0 to 0.056 , which corresponds to stable oscillations of the pendulum around the upper position at the amplitudes up to $0.95 \mathrm{rad}$. Fig. 4 illustrates the convergence of the processes for different initial states of the pendulum when the desired virtual energy $\bar{E}^{*}=0.005$, and the oscillation amplitude is $0.22 \mathrm{rad}$.

\section{CONCLUSION}

Models of Kapitza pendulum and their properties were analyzed by using the concept of virtual energy. The problem of stabilization of a given periodic motion of the pendulum around the upright position was reduced to stabilization of the virtual energy and solved by standard techniques of energy-based control. In order to estimate the slow variables of the system and its virtual energy, a nonlinear observer of the vibrating pendulum was designed. The simulations confirmed the validity of the results. 

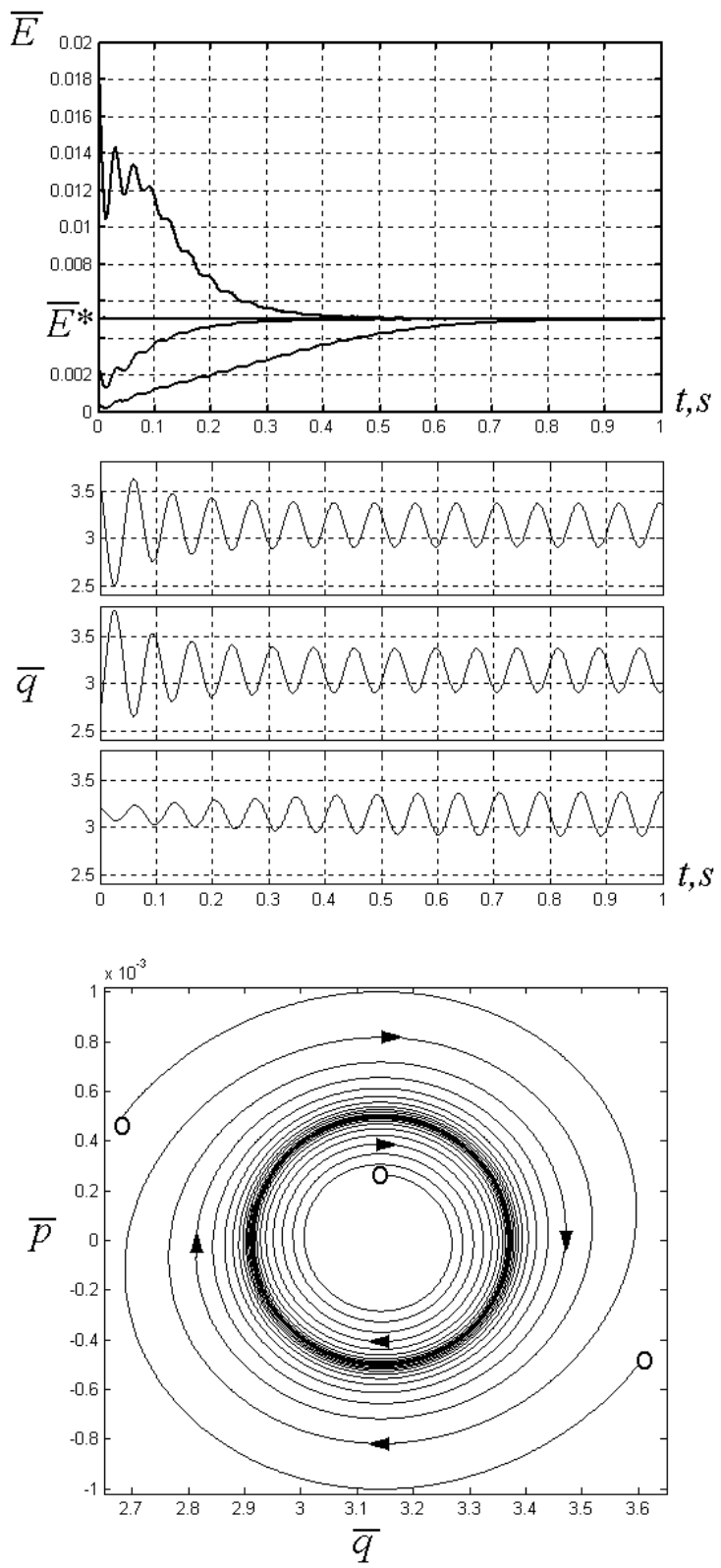

Fig. 4. Stabilization of oscillations around upper position

\section{REFERENCES}

Andrievsky, B.R., P.Yu. Guzenko and A.L. Fradkov (1996). Control of nonlinear oscillations of mechanical systems by speed-gradient method. Automation and Remote Control 4, 4-17.

Aracil, J., F. Gordillo and J.A. Acosta (2002). Stabilization of oscillations in the inverted pendulum. In: 15 IFAC World Congress.

Belman, R.E., J. Bentsman and S.M.Meerkov (1986). Vibrational control of a class of nonlinear systems: Vibrational stabilization. IEEE Trans. Aut. Control 32(8), 710-716.

Blekhman, I. (1988). Synchronization in Science and Technology. ASME Press. New York.

Bogolyubov, N. and Ju.A. Mitropolsky (1962). Asymptotic methods in theory of nonlinear oscillations. Pfismatgiz. Moscow (in Russian).

Chung, C.C. and J. Hauser (1995). Nonlinear control of a swinging pendulum. Automatica 31, 851-862.

Fradkov, A.L. (1996). Swinging control of nonlinear oscillations. International Journal of Control 64(6), 1189-1202.

Fradkov, A.L. and A. Pogromsky (1998). Introduction to control of oscillations and chaos. World Scientific. Singapore.

Fradkov, A.L., I.V. Miroshnik and V.O. Nikiforov (1999). Nonlinear and Adaptive Control of Complex Systems. Kluwer Acad. Pub. Dordrecht.

Kapitza, P.L. (1951). Dynamical stability of a pendulum when its point of suspension vibrates and pendulum with a vibrating suspension. In Collected papers of P.L. Kapitza 2D, Tar Harr, Pergamon Press Ltd.. London.

Miroshnik, I. and A. Bobtzov (2000). Stabilization of motions of multipendulum systems. In: 2nd Int. Conf. on Control of oscillation and chaos. Vol. 1. pp. 22-25. St.Petersburg.

Miroshnik, I.V and E. Olkhovskaya (2003). Spatial problems of nonlinear dynamics. motivation and analysis. In: 2003 Int. Conference Physics and Control. Proceedings. Vol. 2. pp. 582-588. St.Petersburg.

Miroshnik, I.V and N. Odinets (2004). Stabilization of pendulum oscillations around upper position. In: 6th IFAC Syposium on Nonlinear Control Systems (NOLCOS 2004). Vol. 3. pp. 1349-1354. Stuttgart.

Odinets, N. and N. Levidova (2004). Kapitsa pendulum: Modelling and observing. In: Preprints of 10-th Baltic Olympiad on Automatic Control. pp. 22-28. SPbSU ITMO, St.Petersburg.

Shiriaev, A.S., O. Egeland and H. Ludvigsen (1998). Global stabilization of unstable equilibrium point of pendulum. In: Proc. 37th CDC. pp. 4584-4585. Tampa.

Stephenson, A. (1908). On induced stabily. Phil. Mag. 15, 233-236.

Yabuno, H., K. Goto and N. Aoshima (2004). Swing-up and stabilization of an underactuated manipulator without state feedback of free joint. IEEE trans. Robotics and Automation 20, 259-365. 